\title{
Research of underwater sensor network routing protocol based on the depth
}

\author{
Yanping Cong ${ }^{1, a}$,Xiao $\mathrm{Li}^{1, \mathrm{~b}}$,Mengdie $\mathrm{Xu}^{2}$ and Bo Yin ${ }^{1}$ \\ ${ }^{1}$ Ocean University of China, Qingdao 266000, China; \\ ${ }^{2}$ School of Government Beijing Normal University, Beijing 100875, China; \\ acongyp@163.com, ${ }^{b}$ lixiao_uni@163.com
}

Keywords: Underwater sensor network, Depth ,Routing protocol ,Energy consumption

\begin{abstract}
Underwater sensor network(USN) has a battery replacement, positioning difficult hard, great depth of nodes is poor, traditional routing algorithm of the two-dimensional plane land web is difficult to apply, routing algorithm based on the geographical position, there is also a difficult position and so on characteristics. Based on the above reasons, the existing underwater sensor network routing algorithm based on depth, on the basis of this paper proposes a shake hands first, then send the datagram routing protocol, effectively reduce a lot of redundant data to send, so as to effectively reduce the energy consumption.
\end{abstract}

\section{Introduction}

Around the national Marine resources development, the research of USN is more and more attention. Underwater wireless communication network research began in the 90s of last century, in the literature [1], the university of Connecticut underwater acoustic network laboratory study of USN. Research on USN in China starts late, underwater routing algorithm is one of the core problems of underwater sensor network research.

Compared with terrestrial sensor networks, the particularity of USN make traditional routing algorithms cannot be applied to then USN. These differences include the following: first of all in terrestrial communication using electromagnetic waves, and electromagnetic wave attenuation underwater, distortion is serious, general underwater communication using underwater acoustic communication technology. With the extension of distance, the underwater acoustic signal attenuation. Second, the traditional wireless sensor network routing protocol is the assumption that the sensor nodes distributed in a lot of a two-dimensional plane, but ignore its height. And underwater sensor is influenced by ocean currents, waves, move more frequently. These differences are requirements we must according to the characteristics of the underwater communication, to design a kind of special used for USN routing protocol.

\section{Research status and analysis}

Now most of the underwater sensor network routing protocol research method based on geographic location, and based on the $3 \mathrm{~d}$ information to planning the routing node. VBF protocol was proposed in Literature [2], by calculating the forwarding nodes satisfaction factor to adjust the forward delay. This protocol is simple, less amount of calculation. Greedy geographic routing protocol for 3D wireless sensor networks was proposed in Literature [3]. The agreement for the greedy algorithm based on geographic information routing hole problems, which were liable to occur given a $3 \mathrm{D}$ rolling ball boundary calendar calculation method.

Although based on the geographic location of the USN routing protocol efficiency is higher, the price is small, but if using this method, need to get the $\mathrm{x}, \mathrm{y}, \mathrm{z}$ three dimensional information. The $\mathrm{x}, \mathrm{y}$ information is a great research challenges. Generally need to use GPS positioning, due to the underwater GPS signal attenuation is very big, network node is difficult to receive underwater positioning signals, so routing algorithm based on geographical location in the practical feasibility and stability in underwater sensor networks are questionable. But in underwater sensor networks, the 
depth of the sensor error is large, and the depth can be measured by the depth of the sensor. Test all the data in the end to return to the surface. There is some underwater sensor network routing protocol based on depth.

Literature [4] analyzed DBR[5] protocol, the key idea of DBR agreement is all nodes forward packets in the network decision depends on the depth of the node information. When using the greedy forwarding algorithm, node based on certain standards to choose a neighboring node as the next-hop node packets.DBR relative to other location-based information of USN routing protocol, nodes do not need to know the whole $3 \mathrm{D}$ location information, only need to know the local depth of node information. But the DBR there are still some problems, these problems will affect the performance of the network.DBR by flood propagation mechanism, if each node involved in data forwarding, would increase the complexity of the network, have a lot of redundant data, lead to excessive energy consumption, and reduce the network bandwidth utilization.

The methods In this paper inhibit the spread of the flood routing protocol, the sending node first send the message of inquiry to the nodes in it's radius. The sending node get the id and the depth of each node information after each node response, calculate the best forwarding nodes. Fill in the forwarding node id in the data message, so, when each node receives data, only the one to be chosen will forward the datagram. This method suppresses the large number of redundant data transmission. Save energy, improve the utilization of network bandwidth. This protocol also provides a simple solution to the empty cases. when meeting the hole, the node sends a response message to told that it met a hole, not suitable for as the forwarding node. A node after receipt of this message, will choose the second for forwarding node in routing. So that the data will be forward out.

\section{The realization of the protocol based on depth}

The network model. As shown in figure 1, on the surface of the floating multiple sink node, USN messages eventually transferred to the surface of the water, when the surface of the suspension of the sink node receives the message, by ordinary terrestrial wireless network communication data back to the vessel, etc.

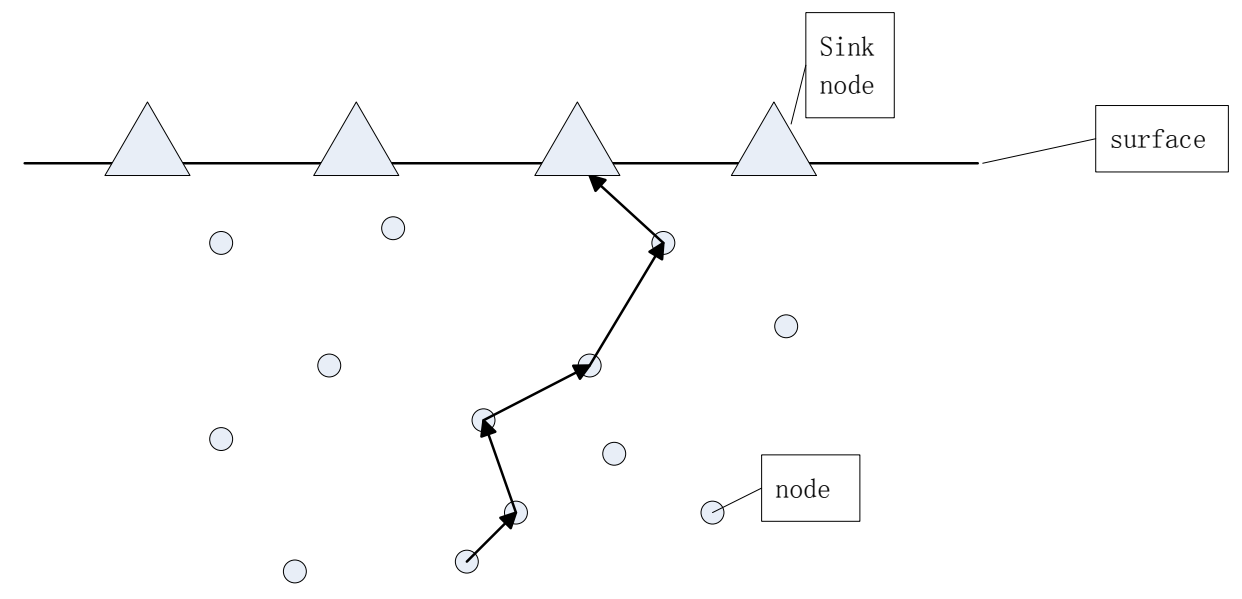

Fig. 1 Node distribution and transmission schematic diagram

Routing algorithm. Firstly, n1 broadcasting explore message, a message to fill in the SID field with own ID, the Depth field to fill their own Depth value, fill status field with 0, indicates that the message to a message. Explore/reply message format shown in table 1, the SID is the sender's ID, the Depth is the Depth of the sender, the Status of 0 said to inquire message, the reply message to 1,2 that message inaccessible. Within the scope of $\mathrm{n} 1$ radio radius $\mathrm{R}$ all nodes will receive this message. When a node receives the message, by examining the message format, determine the message as handshake message, check the Status message segment, if its value is 0, then determine the message 
for inquiry message, then sends a response message. Response message including its ID and depth, as well as the status code 1 .

Table 1 Explore/answer a message and a datagram packet

\begin{tabular}{|l|l|l|}
\hline SI & Dept & Status \\
D & $\mathrm{h}$ & \\
\hline
\end{tabular}

\begin{tabular}{|l|l|l|l|}
\hline Sid & Rid & $\begin{array}{l}\text { Sourcei } \\
\mathrm{d}\end{array}$ & Data \\
\hline
\end{tabular}

When $\mathrm{n} 1$ received each node's response message, update their routing table, routing table format as shown in the table 2. Because in the marine environment, a node by ocean currents, affecting aquatic life will have some movement, of course, also possible that because of the detection node needs to produce autonomy movement. If the depth information of a node changes its depth value is updated. The old routing table that the node information is not included in the latest reply message will be deleted. Thereafter, the node $\mathrm{n} 1$ starts calculating the difference $\mathrm{d}$ between the value of the depth in the routing table and the depth of its own node. $d=d_{n 1}+d_{n x}, d_{n x}$ said depth of all nodes in the routing table. As shown in Fig.2, the node to maximize the $d$ is n3.N1 think that $n 3$ for its transfer point.

Table 2 The routing table

\begin{tabular}{|c|c|c|}
\hline Node ID & Depth & Status \\
\hline $\mathrm{n} 3$ & 56 & 1 \\
\hline $\mathrm{n} 4$ & 89 & 1 \\
\hline$\ldots$ & $\ldots$ & $\ldots$ \\
\hline
\end{tabular}

When you find the furthest up node n3 away from the node n1, n1 generate data packets. Message id field filled n1 node ID, rid field filled n3 of ID, sourceid field filled initially sending node ID. source id field is used to determine the message sink node from which the sensor node.

All nodes within a radius $\mathrm{R}$ range $\mathrm{n} 1$ received the packet. When each node receives the packet, the packet format is judged according to the packet data packets, instead of a handshake packet. This node will extract the purpose ID in the message, and compared with their own ID. If they are same ,the node will do the next step forward. If it finds a different purpose ID with it's own id, it means they do not have the qualifications to forward, then discard the packet.

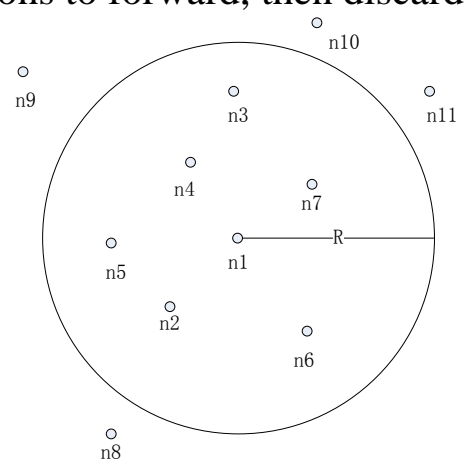

Fig. 2 N1 node and neighbor node schematic

Since the sensor node density can not be large enough that each node can be found to meet the conditions of the transfer point, as shown in Fig. $3 \mathrm{n} 1$ forward radius only $\mathrm{n} 2$ and $\mathrm{n} 6$ and $\mathrm{n} 6$ depth is larger than the $\mathrm{n} 1$, and $\mathrm{n} 1$ forwarded to nodesn2. Found no smaller than the radius within which forwarded it's own node $\mathrm{n} 2$ when forwarding, thus, $\mathrm{n} 2$ will send a reply message to $\mathrm{n} 1$ node. State code reply message is 3 , indicating that unreachable. $\mathrm{n} 1$ node after receiving this message, updates its routing table, the node $\mathrm{n} 2$ marked as unreachable. N1 need to send a message right now, while the depth of the $n 6$ node value is greater than the depth of the $n 2$ node values, but only in its routing table $\mathrm{n} 6$ nodes can be forwarded, so the $\mathrm{n} 1$ node will forward to n6. n6 possibly through n3, n4, n5 forwards the data to the sink node. 


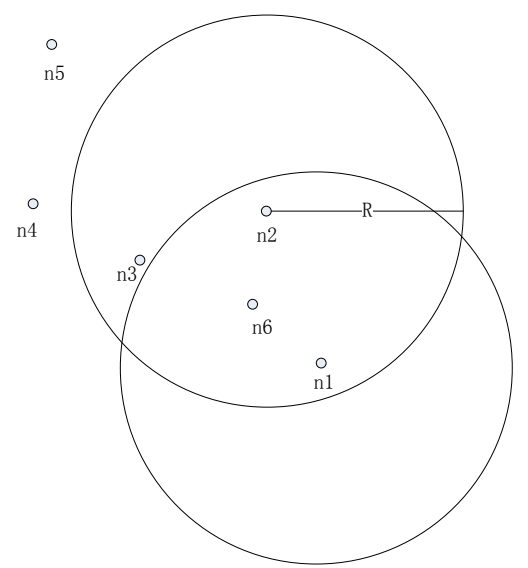

Fig. 3 Schematic empty

\section{Summary}

Based on the special underwater sensor network, this paper proposes an improved routing algorithm based on depth. As a result of underwater sensor networks in energy supply problems, so there are strictly in terms of energy consumption. Routing protocol to shook hands for the depth of the forwarding nodes information, so as to choose the best forwarding nodes. Only the selected node will forward data, so as to avoid a lot of redundant data forwarding, to reduce energy consumption, prolonging network survival time.

\section{Acknowledgement}

This work was financially supported by Qingdao innovation and entrepreneurship leading talent project(13-cx-2),Qingdao strategic industry development project(13-4-1-15-HY) and Shandong province science and technology project(2013GHY11519).

\section{References}

[1] Jun-Hong Cui, Jiejun Kong, Mario Gerla, et al.Challenges: Building scalable mobile underwater wireless sensor networks for aquatic applications [J ].IEEE Network, Special Issue on Wireless Sensor Networking, 2006, 20(3): 12-18.

[ 2] Xie P , Cui J H, Lao L. VBF: Vector -Based forwarding protocol for underwate rSensor networks [ R] .UCONNCSE UbiNet TR 05 -03 Technical Report , 2005.

[3]Mao Jian, Wu Hongyan. Greedy geographic routing protocol for 3D wireless sensor networks. Transducer and Microsystem Technologies, 2011; 30(1): 70-72.

[4] Fen Huang, Chen Ming-song. DBR underwater wireless sensor network routing protocol research [J]. TV Technology,2012,13:74-77.

[5]Yan Hai, Shi Zhijie, CUI Junhong. DBR: depth based routing for underwater sensor network [C ] / /Proc. NETWORKING' 08. [S. l. ] :Springer-Verlag Berlin, 2008:16-1221. 